\title{
OVSF-CDMA Code Assignment in Wireless Ad Hoc Networks
}

\author{
Peng-Jun Wan* Xiang-Yang Li* Ophir Frieder*
}

\begin{abstract}
Orthogonal Variable Spreading Factor (OVSF) CDMA code consists of an infinite number of codewords with variable rates, in contrast to the conventional orthogonal fixed-spreadingfactor CDMA code. Thus, it provides a means of supporting of variable rate data service at low hardware cost. However, assigning OVSF-CDMA codes to wireless ad hoc nodes posts a new challenge since not every pair of OVSF-CDMA codewords are orthogonal to each other. In an OVSF-CDMA wireless ad hoc network, a code assignment has to be conflict-free, i.e., two nodes can be assigned the same codeword or two non-orthogonal codewords if and only if their transmission will not interfere with each other. The throughput (resp., bottleneck) of a code assignment is the sum (resp., minimum) of the rates of the assigned codewords. The maxthroughput (resp., max-bottleneck) conflict-free code assignment problem seeks a conflict-free code assignment which achieves the maximum throughput (resp., bottleneck). In this paper, we present several efficient methods for conflict-free code assignment in OVSF-CDMA wireless ad hoc networks. Each method is proved to be either a constant-approximation for max-throughput conflict-free code assignment problem, or a constant-approximation for max-bottleneck conflictfree code assignment problem, or constant-approximations for both problems simultaneously.
\end{abstract}

Keywords: OVSF-CDMA, code assignment, approximation algorithms, wireless ad hoc networks.

\section{Introduction}

Code division multiple access (CDMA) provides higher capacity, flexibility, scalability, reliability and security than conventional frequency division multiple access (FDMA) and time division multiple access (TDMA). It has already been widely deployed in the second generation cellular communication systems and was proposed for the emerging and future wireless systems, including wireless local area networks, and wireless ad hoc networks. In a CDMA system, the communication channels are defined by the pseudo-random codewords, which are carefully designed to cancel each other out as far as possible. Each communication utilizes the entire available spectrum, and every bit of data is multiplied by the codeword used by the communication channel. Thus, many duplicates of the same information is transmitted to ensure that at least one gets through. The number of duplicates, which is equal to the length of the codeword, is know as the spreading factor. The inverse of the length of the codeword is known as the rate of the codeword. There is a trade-off on the length of the codewords. On one hand, longer codewords can increase the number of available channels and the robustness of the communications. On the other hand, longer codewords would result in lower data rate of the communication channels since the raw data rate seen by the user is the inverse to the codeword length.

${ }^{*}$ Department of Computer Science, Illinois Institute of Technology, Chicago, IL 60616. Emails: \{wan, xli, ophir\}@cs.iit.edu. The work of Peng-Jun Wan and Xiang-Yang Li is partially supported by NSF CCR-0311174. 
Conventional CDMA used for voice communications in the cellular systems is of constant rate in nature. Correspondingly, all codewords in the code have fixed length. Such code is referred to as orthogonal fixed-spreading-factor (OFSF) code. In the past several years, data services have become increasingly important to the cellular networks. Indeed, one major role of the third generation cellular systems is to support differentiated quality-of-service (QoS) guarantees for emerging multimedia applications, which are typically of variable data rate. The support of high-rate data service by OFSF code can be achieved by assigning multiple codewords to a connection. This mode of operation is called multicode CDMA (MC-CDMA). However, MC-CDMA requires multiple transceivers units at each node, thus introduces increased hardware complexity.

Motivated by the support of variable rate data service at low hardware cost, a variable-length code, known as orthogonal variable-spreading-factor (OVSF) code, was developed [1] in 1997. The idea of the OVSF code is to allow the codewords in the code to have variable lengths, and a higherrate request is assigned a single shorter codeword. So by using OVSF code, only a single transceiver is required per node. The generation of OVSF code can be depicted by a code-tree structure [1] shown in Figure 1(a). The code-tree is a balanced binary tree, whose vertices represent the codewords. The root, which is at the level zero, is associated with the codeword 1. Recursively, if a vertex has codeword $c$, then its two children have codewords $c c$ and $c \bar{c}$ respectively, where $\bar{c}$ is the complement of $c$. Thus, at level $l$ there are $2^{l}$ codewords, each $2^{l}$ bits long. To simplify our notations, we represent the $i$ th OVSF-CDMA codeword at layer $l$ by a $l+1$-bits binary representation, where the most significant bit is always 1 and the remaining $l$ bits are the binary representation of of integer $i-1$. For example, the 1st codeword at layer 3 of the codeword tree is 10101010, which we will denote as 1000 in our binary representation; the 3rd codeword at layer 3 of the codeword tree is 10011001, which we will denote as 1010 in our binary representation. See Figure 1(b) for the corresponding binary color representation of the CDMA codewords.

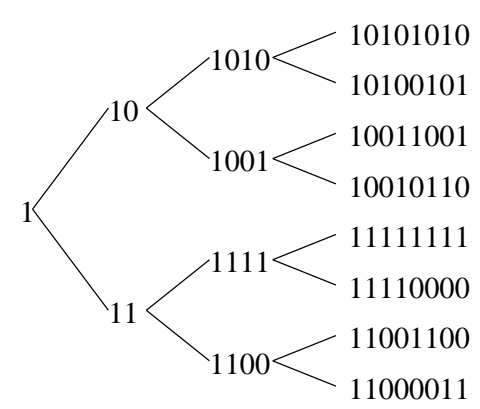

(a)

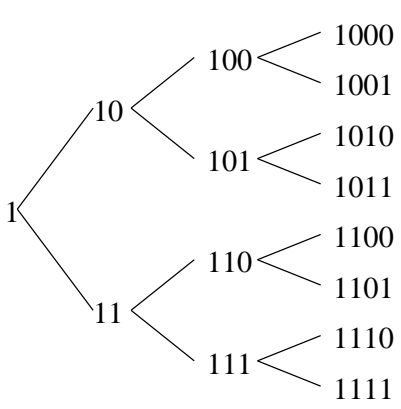

(b)

Figure 1: OVSF code: (a) code-tree structure; (b) binary color representation.

OVSF code has two prominent features different from OFSF code: (1) The number of the codewords in an OVSF code is infinity, while the number of codewords in an OFSF code is finite. (2) Not every pair of codewords in an OVSF code are orthogonal to each other. Indeed, two OVSF codewords are orthogonal to each other if and only neither is an ancestor, or equivalently, a prefix of the other. On the other hand, all codewords in an OFSF code are orthogonal to each other.

A wireless ad hoc network is a collection of radio nodes (transceivers) located in a geographic region. Each node is equipped with an omnidirectional antenna and has limited transmission power. A communication session is established either through a single-hop radio transmission if 
the communication parties are close enough, or through relaying by intermediate nodes otherwise. A channel assignment to the nodes in a wireless as hoc should avoid two collisions. The primary collision occurs when a node simultaneously transmits and receives signals over the same channel, or two non-orthogonal channels in case of OVSF-CDMA. The secondary collision occurs when a node simultaneously receives more than one signals over the same channel, or non-orthogonal channels in case of OVSF-CDMA. Thus, to prevent the primary collision, two nodes can be assigned the same channel or two non-orthogonal channels if and only if neither of them is within the transmission range of the other. Similarly, to prevent the secondary collision, two nodes can be assigned the same channel or two non-orthogonal channels if and only if no other node is located in the intersection of their transmission ranges.

Given an OFSF-CDMA code assignment, its throughput is the sum of the rates of the assigned codewords, and its bottleneck is the minimum of the rates of the assigned codewords. The throughput of a wireless ad hoc network is then the maximum of the throughput over all possible conflict-free OFSF-CDMA code assignment to its nodes. Similarly, the bottleneck of a wireless ad hoc network is then the maximum of the bottleneck over all possible conflict-free OFSF-CDMA code assignment to its nodes. In this paper, we will study the OVSF-CDMA code assignment for wireless ad hoc networks with objectives to maximize the throughput of the assignment or the bottleneck of the assignment or both.

The main contributions of this paper are as follows. We first establish the relation between the independence number and the throughput, and the relation between the bottleneck and the chromatic number. We show that the throughput of the optimum CDMA code assignment for a wireless ad hoc network is at least $\frac{5}{8}$ of the independence number of the corresponding interference graph. The bottleneck of the optimum CDMA code assignment for a wireless ad hoc network is at least a small constant factor of the chromatic number of the corresponding interference graph. After that we present several heuristics for conflict-free OVSF-CDMA codeword assignment. The obtained code assignments can achieve a throughput within a constant factor of the maximum throughput, and/or a bottleneck within a constant factor of the maximum bottleneck. We also extend our results to a more general wireless ad hoc network model where each wireless node has an interference disk inside which its signal will interfere, and it has a transmission disk inside which a node can receive its signal correctly.

It will be seen that the correctness of the methods presented in this paper does not require that the transmission region of each wireless device is a disk centered at this node. Out methods apply to all wireless networks when the communication channels are varying with distance, with time, and with obstacles. The specific wireless network model used here only enables us to prove that our methods have theoretical performance guarantees. The correctness of our methods also do not depend on the node positions. The usage of the node positions enables us to design algorithms with better approximation ratios. The position error will not affect our methods as long as the position error will not change the topology of the network, i.e., the network topology derived from the perceived nodes' positions is the same as the actual physical network topology.

The remainder of the paper is organized as follows. In Section 2, we provide a graph-theoretical formulation of the conflict-free code assignment problems in wireless ad hoc networks and briefly review the related works. In this section, we also prove a key technical lemma which will be used later in the paper. In Section 3, we establish the relation between the independence number and the throughput, and the relation between the bottleneck and the chromatic number. In Section 4, we propose several heuristics for conflict-free code assignment and analyze their performances. Finally, we conclude our paper in Section 5. 


\section{Preliminaries and Related Works}

\subsection{Problems Formulation}

Let $V$ be the set of radio nodes in a given wireless ad hoc network, and $r_{v}$ be the specified transmission radius of node $v$ for each $v \in V$. For any pair of nodes $u$ and $v$, we use $\|u v\|$ to denote their Euclidean distance. Then a geometric graph $G$ over $V$ can be obtained by creating an edge between each pair of nodes $(u, v)$ satisfying that either $\|u v\| \leq \max \left\{r_{u}, r_{v}\right\}$ or there is a node $w \in V \backslash\{u, v\}$ such that $\|u w\| \leq r_{u}$ and $\|v w\| \leq r_{v}$. The graph $G$ is referred to as the interference graph.

With the introduction of the interference graph, a conflict-free channel assignment in wireless ad hoc networks channelized by FDMA, TDMA, or OFSF-CDMA, is equivalent to a proper vertex coloring of the interference graph. However, such equivalency disappears if the wireless ad hoc network is channelized by OVSF-CDMA. Instead, a conflict-free channel assignment in a wireless adhoc network channelized by OVSF-CDMA is equivalent to the following variant of vertex coloring, referred to as prefix-free vertex coloring, or simple prefix-free coloring, of the interference graph $G$ : The colors are represented by positive binary numbers as shown in Figure 1(b). Note that the first (i.e., leftmost) bit of every binary color is one, and a binary color at level $l$ has $l+1$ bits. Two binary colors are said to be prefix-free if neither is a prefix of the other. Then, two binary colors are prefix-free if and only if the corresponding codewords are orthogonal. A prefix-free coloring of $G$ is a vertex coloring such that any pair of adjacent vertices in $G$ receive prefix-free colors.

We associate each binary color with a rate attribute, which is equal to the rate of the corresponding codeword. Thus, the rate of an $i$-bit binary color is equal to the $2^{-i+1}$. Given a conflict-free CDMA/OVSF code assignment $\left\{c_{v} \mid v \in V, \forall\right.$ link $u v, c_{u}$ and $c_{v}$ are orthogonal $\}$ of the interference graph $G$, its throughput and bottleneck are defined as $\sum_{v \in V} 2^{-\left|c_{v}\right|+1}$ and $\min _{v \in V} 2^{-\left|c_{v}\right|+1}$ respectively, where $\left|c_{v}\right|$ denotes the number of bits of the color $c_{v}$. In other words, the throughput of a conflict-free CDMA/OVSF code assignment is the sum of the rates of the assigned codes, and its bottleneck is the minimum of the rates of the assigned codes. The throughput of an interference graph $G$, denoted by $\tau(G)$, is then the maximum of the throughput over all possible conflict-free CDMA/OVSF code assignments of $G$. Similarly, the bottleneck of an interference graph $G$, denoted by $\beta(G)$, is then the maximum of the bottleneck over all conflict-free CDMA/OVSF code assignments of $G$.

\subsection{Related Works}

All prior studies of prefix-free coloring have been restricted to complete graphs in the context of channel assignment to nodes in a single cell of an OVSF-CDMA cellular networks $[3,5,10,16]$. The prefix-free vertex coloring of complete graphs is fairly easy. Indeed, since each node must receive a unique color different from others, a prefix-free coloring can thus be represented by a binary tree with one-to-one correspondence between the nodes (or their colors) and the leaves. Every binary tree with $n$ leaves leads to a valid prefix-free coloring. If the binary tree is full, then the corresponding coloring achieves the maximum throughput one. If the binary tree is full and balanced, the corresponding coloring achieves both maximum throughput and maximum bottleneck. Furthermore, if each node specifies a demand equal to a power of $1 / 2$, then as an immediate application of Kraft's inequality, all demands can be satisfied if and if the total demands is at most one. The dynamic reassignment of colors to meet a new demand is addressed in [16].

The minimum (proper) vertex coloring of the interference graph have been studied in the context of channel assignment in wireless ad hoc networks channelized by FDMA, TDMA or OFSF-CDMA 
$[6,7,9,11,17,18,19,20,21,22,23]$. The majority of these works simply presented networking protocols to obtain a proper coloring without addressing the computational complexity or the theoretical performance. Sen and Huson [20] proved the NP-hardness the minimum vertex coloring of the interference graph even when all nodes are located in a plane and have the same transmission radii. Sen and Malesinska [21] made an attempt to analyze the approximation ratio of the classical FIRST-FIT coloring in smallest-degree-last ordering due to Matula and Beck [15] when applied to the interference graph. Unfortunately, their analysis turned to be erroneous. Wan et al. [23] recently provided correct and tighter analysis of Matula and Beck's algorithm and several other approximation algorithms as well.

A problem related to the vertex coloring of the interference graphs is the distance-2 vertex coloring of a graph [12]. A distance-2 vertex coloring of a graph $G$ is a coloring of the vertices such that any two vertices separated by at most two hops receive different colors. In other words, it is a proper vertex coloring of $G^{2}$, the square graph of $G$-the graph obtained by creating an edge between each pair of vertices of $G$ whose graph distance in $G$ is at most two. When all nodes have equal transmission radii, their interference graph happens to be the square of unit-disk graph over these nodes, and hence in this case, the vertex coloring of the interference graph is the same as a distance-2 vertex coloring of a unit-disk graph [8]. However, when the nodes have disparate transmission radii, the interference graph may be not the square of any graph as observed in [23]. Therefore, distance-2 vertex coloring is in general different from the vertex coloring of the interference graphs.

To our best knowledge, there has been no attempt to maximize the throughput when coloring vertices. The only vertex coloring problem that can be considered to be somehow related is the minimum chromatic sum problem $[4,13]$, which seeks a vertex coloring of a given graph $G$, using natural numbers, such that the total sum of the colors of the vertices is minimized among all proper vertex coloring of $G$. However, the maximum-throughput prefix-free vertex coloring problem possesses several unique features, which makes itself different from the minimum chromatic sum problem. First of all, the vertex coloring must be prefix-free, instead of being proper only. Second, the rate of the colors is different from the color number itself. Third, it is the maximization problem, while the minimum chromatic sum problem is a minimization problem.

\subsection{A Technical Lemma}

In this subsection, we will prove some important lemmas which will later be used to study some fundamental properties of an optimum OVSF-CDMA code assignment for wireless ad hoc networks.

Let $T$ be a (rooted) binary tree. For each vertex $v$ of $T$, the level of $v$ in $T$, denoted by $\ell_{T}(v)$ is defined as the length of the path in $T$ between the root and $v$. Thus the level of the root is zero. A binary tree is full if every non-leaf vertex has exactly two children. A binary tree is balanced if the levels of all leaves differ by at most one. A binary tree is said to be extremely unbalanced if there are exact two leaves at the maximum level and one leaf at any other level (see Figure 2).

Consider a finite set $S$ of items in which each item $s$ is associated with a positive weight $\omega(s)$. Let $\mathcal{T}_{S}$ denote the set of binary trees whose leaves are the items of $S$. For each tree $T$ in $\mathcal{T}_{S}$, its throughput, denoted by $f(T)$, is defined by

$$
f(T)=\sum_{s \in S} \omega(s) 2^{-\ell_{T}(s)} .
$$

A tree in $\mathcal{T}_{S}$ is said to be optimal if its throughput achieves the maximum among all trees in $\mathcal{T}_{S}$. Obviously, any optimal tree must be full. Let $T^{*}$ be an extremely unbalanced tree in $\mathcal{T}_{S}$ satisfying 


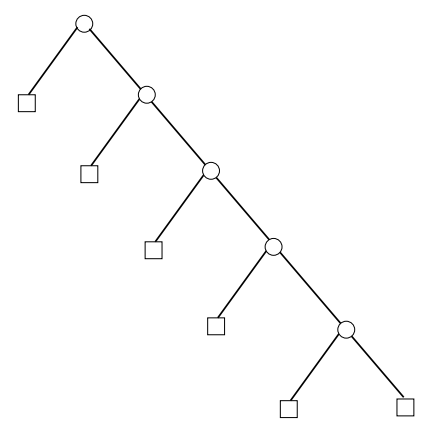

Figure 2: An extremely imbalanced full binary tree.

that the levels of the items sorted in the decreasing order of the weights monotonically increase. The next lemma states that $T^{*}$ is optimal.

Lemma $1 T^{*}$ is an optimal tree in for $\mathcal{T}_{S}$. If $S$ is a finite set of items with weights $\omega_{1} \geq \omega_{2} \geq$ $\cdots \geq \omega_{k}$, then its throughput is

$$
\sum_{i=1}^{k-1} \frac{\omega_{i}}{2^{i}}+\frac{\omega_{k}}{2^{k-1}} .
$$

The proof of this lemma is similar to the proof of the correctness of Huffman code construction (see, e.g., Chapter 16 of [2]). It will use the following two lemmas.

Lemma 2 Let $x$ and $y$ be two items having the lowest weights. Then there exists an optimal tree in which $x$ and $y$ appear as the sibling leaves of maximum level.

Proof. The idea of the proof is to take an arbitrary optimal tree $T$ and modify it to make a tree representing another optimal tree such that $x$ and $y$ appear as the sibling leaves of maximum level in the new tree. We use the swapping argument. Let $a$ and $b$ be two items that are sibling leaves of the maximum level in $T$ (see Figure 3). Without loss of generality, we assume that $\omega(x) \leq \omega(y)$ and $\omega(a) \leq \omega(b)$. Then $\omega(x) \leq \omega(a)$ and $\omega(y) \leq \omega(b)$. As shown in Figure 3, we exchange the
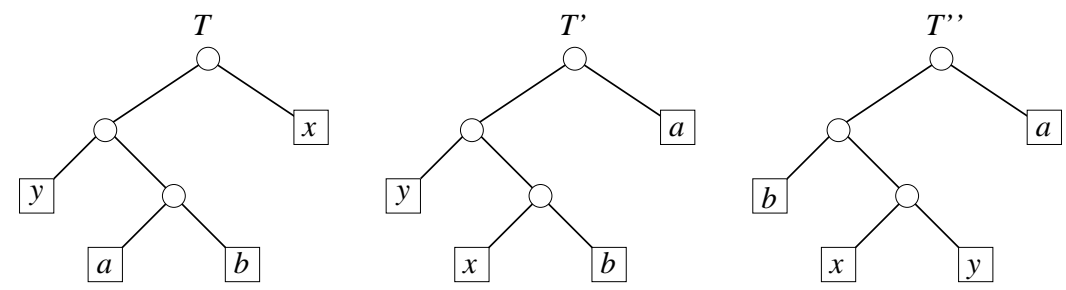

Figure 3: An illustration of the swap operations in the proof of Lemma 2.

positions in $T$ of $a$ and $x$ to produce a tree $T^{\prime}$, and then we exchange the positions in $T^{\prime}$ of $b$ and 
$y$ to produce a tree $T^{\prime \prime}$. The difference in throughput between $T$ and $T^{\prime}$ is

$$
\begin{aligned}
f(T)-f\left(T^{\prime}\right) & =\sum_{s \in S} \omega(s) 2^{-\ell_{T}(s)}-\sum_{s \in S} \omega(s) 2^{-\ell_{T^{\prime}}(s)} \\
& =\omega(x) 2^{-\ell_{T}(x)}+\omega(a) 2^{-\ell_{T}(a)}-\omega(x) 2^{-\ell_{T^{\prime}}(x)}-\omega(a) 2^{-\ell_{T^{\prime}}(a)} \\
& =\omega(x) 2^{-\ell_{T}(x)}+\omega(a) 2^{-\ell_{T}(a)}-\omega(x) 2^{-\ell_{T}(a)}-\omega(a) 2^{-\ell_{T}(x)} \\
& =(\omega(a)-\omega(x))\left(2^{-\ell_{T}(a)}-2^{-\ell_{T}(x)}\right) \\
& \leq 0
\end{aligned}
$$

because $\omega(a) \geq \omega(x)$ and $\ell_{T}(a) \geq \ell_{T}(x)$. Thus, $f(T) \leq f\left(T^{\prime}\right)$, which means exchanging $x$ and $a$ does not decrease the throughput. Similarly, exchanging $y$ and $b$ does not decrease the throughput and hence $f\left(T^{\prime}\right) \leq f\left(T^{\prime \prime}\right)$. Therefore, $f(T) \leq f\left(T^{\prime \prime}\right)$. Since $T$ is optimal, $f(T)=f\left(T^{\prime \prime}\right)$. Thus, $T^{\prime \prime}$ is an optimal tree in which $x$ and $y$ appear as the sibling leaves of maximum level, from which the lemma follows.

The next lemma shows that the optimal tree has the optimal-substructure property.

Lemma 3 Let $T$ be an optimal tree in $\mathcal{T}_{S}$. Consider any two items $x$ and $y$ that appear as the sibling leaves in $T$, and let $z$ be its parent. Then, considering $z$ as an item with weight $\omega(z)=\frac{\omega(x)+\omega(y)}{2}$, the tree $T^{\prime}$ obtained from $T$ by putting $z$ at the parent of $a$ and $y$ and them removing $x$ and $y$ is optimal tree in $\mathcal{T}_{S^{\prime}}$ where $S^{\prime}=S-\{x, y\} \cup\{z\}$.

Proof. We first show that the throughput $f(T)$ of $T$ is equal to the throughput $f\left(T^{\prime}\right)$ of $T^{\prime}$. For each $s \in S-\{x, y\}$, we have $\ell_{T}(s)=\ell_{T^{\prime}}(s)$ and hence $\omega(s) 2^{-\ell_{T}(s)}=\omega(s) 2^{-\ell_{T^{\prime}}(s)}$. Since

$$
\ell_{T}(x)=\ell_{T}(y)=\ell_{T^{\prime}}(z)+1,
$$

we have

$$
\begin{aligned}
\omega(x) 2^{-\ell_{T}(x)}+\omega(y) 2^{-\ell_{T}(y)} & =(\omega(x)+\omega(y)) 2^{-\ell_{T^{\prime}}(z)-1} \\
& =2 \omega(z) 2^{-\ell_{T^{\prime}}(z)-1} \\
& =\omega(z) 2^{-\ell_{T^{\prime}}(z)}
\end{aligned}
$$

Thus, we conclude that $f(T)=f\left(T^{\prime}\right)$.

If $T^{\prime}$ is not an optimal one in $\mathcal{T}_{S^{\prime}}$, then there exists a tree $T^{\prime \prime}$ in $\mathcal{T}_{S^{\prime}}$ such that $f\left(T^{\prime \prime}\right)>f\left(T^{\prime}\right)$. Since $z$ is treated as an item in $S^{\prime}$, it appears as a leaf in $T^{\prime \prime}$. If we add $x$ and $y$ as children of $z$ in $T^{\prime \prime}$, then we obtain a tree in $\mathcal{T}_{S}$ with $f\left(T^{\prime \prime}\right)>f\left(T^{\prime}\right)=f(T)$, contradicting the optimality of $T$. Thus, $T^{\prime}$ must be optimal in $\mathcal{T}_{S^{\prime}}$.

Note that if $x$ and $y$ are the two items having the lowest weights, then the new item $z$ has the lowest weight in the set $S^{\prime}$. This fact, together with the above two lemmas, implies the correctness of Lemma 1.

\section{Throughput And Bottleneck of General Graphs}

The results in this section holds for general graphs. The concepts of prefix-free coloring, throughput and bottleneck can be extended to general graphs. Let $G$ be an arbitrary graph. Following the standard notations, we use $\chi(G)$ and $\alpha(G)$ to denote the chromatic number and the independence 
number respectively of $G$. We also introduce two new notations. For any graph $G$, we use $\tau(G)$ and $\beta(G)$ to denote the throughput and bottleneck respectively of $G$. The main result of this section is the following relations among these four graph parameters.

Theorem 4 For any graph $G$,

$$
\begin{aligned}
\alpha(G) / 2 & \leq \tau(G) \leq \alpha(G) \\
\beta(G) & =2^{-\lceil\log \chi(G)\rceil} .
\end{aligned}
$$

The proof of the first part of Theorem 4 involves a new concept of canonical prefix-free coloring, which is defined below. We observe that in any prefix-free coloring of $G$, all nodes receiving the same color form an independent set of $G$. Thus, any prefix-free coloring of $G$ can be regarded as a partition of $V(G)$ into independent sets $V_{1}, V_{2}, \cdots, V_{k}$ followed by an assignment of colors to these independent sets as a whole. A prefix-free coloring of $G$ is said to be canonical if it partitions of $V(G)$ into independent sets $V_{1}, V_{2}, \cdots, V_{k}$ with

$$
\left|V_{1}\right| \geq\left|V_{2}\right| \geq \cdots \geq\left|V_{k}\right|
$$

for some integer $k$, and assigns the color $1^{i} 0$ to all nodes in $V_{i}$ for $1 \leq i \leq k-1$ and the color $1^{k}$ to all nodes in $V_{k}$. By definition, a canonical prefix-free coloring is fully determined by the partition of $V$ into independent sets. The next lemma states that there exists an canonical prefix-free coloring of $G$ which achieves the maximum throughput.

Lemma 5 For any graph $G$, there is a canonical prefix-free coloring of $G$ which achieves the maximum throughput.

Proof. A prefix-free coloring which uses $k$ different colors $c_{1}<c_{2}<\cdots<c_{k}$ is said to be locally tight if each node receiving a color $c_{i}$ for some $i>1$ has at least one neighbor receiving the color $c_{j}$ for any $1 \leq j<i$. It is easy to see that every prefix-free coloring can be transformed to a locally tight one with the same or smaller throughput. Therefore, there is a prefix-free coloring which is locally tight and achieves the maximum throughput. Let $O P T$ be a such prefix-free coloring. Assume that $O P T$ uses $k$ different colors $c_{1}<c_{2}<\cdots<c_{k}$. Since $O P T$ is locally tight, these $k$ colors are pairwise prefix-free. For each $1 \leq i \leq k$, let $V_{i}$ denote the set of vertices which receive the color $c_{i}$. Then the $k$ subsets $V_{1}, V_{2}, \cdots, V_{k}$ form a partition of $V(G)$ into independent sets. Now we renumber them such that

$$
\left|V_{1^{*}}\right| \geq\left|V_{2^{*}}\right| \geq \cdots \geq\left|V_{k^{*}}\right|
$$

Let $O P T^{*}$ be the prefix-free coloring which assigns the color $1^{i} 0$ to all nodes in $V_{i^{\prime}}$ for $1 \leq i \leq k-1$ and the color $1^{k}$ to all nodes in $V_{k^{\prime}}$. Then $O P T^{*}$ is a canonical prefix-free coloring. We shall prove that the throughput of $O P T^{*}$ also achieves the maximum throughput by using Lemma 1.

In order to apply Lemma 1 , we treat each subset $V_{i}$ as an item with weight $\omega\left(V_{i}\right)=\left|V_{i}\right|$ and let $S=\left\{V_{1}, V_{2}, \cdots, V_{k}\right\}$. We define two trees $T$ and $T^{*}$ in $\mathcal{T}_{S}$ as follows. For each $1 \leq i \leq k$, let $P_{i}$ denote the path in the tree representation of binary colors shown in Figure 1 from the root to the tree vertex representing color $c_{i}$. Since the $k$ colors $c_{1}, c_{2}, \cdots, c_{k}$ are pairwise prefix-free, the union of the $k$ paths $c_{1}, c_{2}, \cdots, c_{k}$ is a binary tree with $k$ leaves. For each $1 \leq i \leq k$, we place the item $V_{i}$ to the leaf which comes from $P_{i}$. The resulting tree in $\mathcal{T}_{S}$ is then defined to be the tree $T$. The tree $T^{*}$ is defined as the extremely unbalanced binary tree in $\mathcal{T}_{S}$ with the item $V_{i^{*}}$ being the (unique) leaf at level $i$ for each $1 \leq i \leq k-2$ and the two items $V_{(k-1)^{*}}$ and $V_{k^{*}}$ being the two leaves at 
level $k-1$. Clearly, $f(T)$ equals to the throughput of $O P T$, and $f\left(T^{*}\right)$ equals to the throughput of $O P T^{*}$. By Lemma 1, $f(T) \leq f\left(T^{*}\right)$. Thus, the throughput of $O P T$ is less than or equal to the throughput of $O P T^{*}$. Since $O P T$ achieves the maximum throughput, so does $O P T^{*}$.

Proof of Theorem 4: Now we are ready to prove the first part of Theorem 4. First, we show that $\tau(G) \leq \alpha(G)$. Consider a canonical prefix-free coloring of $G$ which achieves the maximum throughput $\tau(G)$. Assume that $k$ colors are used. For each $1 \leq i \leq k$, let $V_{i}$ be the set of nodes receiving the color $1^{i} 0$. Then,

$$
\alpha(G) \geq\left|V_{1}\right| \geq\left|V_{2}\right| \geq \cdots \geq\left|V_{k}\right|
$$

Thus,

$$
\begin{aligned}
\tau(G) & =\sum_{i=1}^{k-1} \frac{\left|V_{i}\right|}{2^{i}}+\frac{\left|V_{k}\right|}{2^{k-1}} \\
& \leq \alpha(G)\left(\sum_{i=1}^{k-1} \frac{1}{2^{i}}+\frac{1}{2^{k-1}}\right) \\
& =\alpha(G) .
\end{aligned}
$$

Second, we prove that $\alpha(G) / 2 \leq \tau(G)$. Let $V_{1}$ be a maximum independent set, and $\left\{V_{2}, \cdots, V_{k}\right\}$ be an arbitrary partition of $V \backslash V_{1}$ into independent sets with

$$
\left|V_{2}\right| \geq \cdots \geq\left|V_{k}\right|
$$

Then,

$$
\alpha(G)=\left|V_{1}\right| \geq\left|V_{2}\right| \geq \cdots \geq\left|V_{k}\right| .
$$

Consider the canonical prefix-free coloring of $G$ determined by $V_{1}, V_{2}, \cdots, V_{k}$. Its throughput is

$$
\sum_{i=1}^{k-1} \frac{\left|V_{i}\right|}{2^{i}}+\frac{\left|V_{k}\right|}{2^{k-1}} \geq \frac{\left|V_{1}\right|}{2}=\frac{\alpha(G)}{2} .
$$

Therefore,

$$
\tau(G) \geq \frac{\alpha(G)}{2}
$$

Next we prove the second part of Theorem 4 . First, we show that $\beta(G) \leq 2^{-\lceil\log \chi(G)\rceil}$. Consider any prefix-free coloring with maximum bottleneck $\beta(G)=2^{-\ell+1}$ for some $\ell$. Then every color in this coloring is at most $\ell$-bit long. We replace each $\ell^{\prime}$-bit color $c$ with $\ell^{\prime}<\ell$ by the $\ell$-bit color $c 0^{\ell-\ell^{\prime}}$, i.e. the color obtained from $c$ by appending $\ell-\ell^{\prime}$ zeros. This new coloring remains prefix-free and uses only $\ell$-bit colors. Since the first bit of every $\ell$-bit color is always one, the total number of $\ell$-bit colors is at most $2^{\ell-1}$. Thus $\chi(G) \leq 2^{\ell-1}$. This implies that $\lceil\log \chi(G)\rceil \leq \ell-1$. Thus,

$$
\beta(G)=2^{-(\ell-1)} \leq 2^{-\lceil\log \chi(G)\rceil} .
$$

First, we show that $\beta(G) \geq 2^{-\lceil\log \chi(G)\rceil}$. Consider any proper vertex coloring of $G$ using $\chi$ colors. These $\chi$ colors can all be represented by distinct $(1+\lceil\log \chi(G)\rceil)$-bit binary colors. Thus,

$$
\beta(G) \geq 2^{-(1+\lceil\log \chi(G)\rceil)+1}=2^{-\lceil\log \chi(G)\rceil} .
$$


This completes the proof of Theorem 4 .

Theorem 4 concentrates on constructing one maximal independent set from the interference graph. Intuitively, if we also construct a good maximal independent set for the remaining nodes, we could improve the performance bound on the throughput of the CDMA code assignment. The new approach will compute a maximal independent $V_{1}^{\prime}$, and then compute a maximal independent $V_{2}^{\prime}$ for the remaining nodes after removing $v_{1}^{\prime}$ from $G$. The nodes in $V_{1}^{\prime}$ will receive a CDMA code 10 and the nodes in $V_{2}^{\prime}$ will receive a CDMA code 110. The following theorem shows that it indeed improves the approximation ratio.

Theorem 6 An @-approximation algorithm for the maximum independent set gives a $\frac{5}{8} \varrho$-approximation algorithm for the maximum throughput CDMA code assignment.

Proof. Consider a canonical maximum independent decomposition $V_{1}, V_{2}, \cdots, V_{k}$ of all nodes $V$. Here $\left|V_{1}^{\prime}\right| \geq \varrho \cdot\left|V_{1}\right|$. Let $t_{i, j}=\frac{\left|V_{i}^{\prime} \cap V_{j}\right|}{\left|V_{j}\right|}$, i.e., the portion of $V_{j}$ is used in $V_{i}^{\prime}$. After $V_{1}^{\prime}$ is generated, we know that the maximum independent set in the remaining graph (be removing $V_{1}^{\prime}$ and all its incident edges) has size at least

$$
\max \left(\left(1-t_{1,1}\right) \cdot\left|V_{1}\right|,\left(1-t_{1,2}\right) \cdot\left|V_{2}\right|\right),
$$

since $V_{1}-V_{1}^{\prime} \cap V_{1}$ and $V_{2}-V_{1}^{\prime} \cap V_{2}$ are still independent sets. Notice that $t_{1,1} \cdot\left|V_{1}\right|+t_{1,2} \cdot\left|V_{2}\right| \leq V_{1}$. Then $\left(1-t_{1,1}\right) \cdot\left|V_{1}\right|+\left(1-t_{1,2}\right) \cdot\left|V_{2}\right| \geq\left|V_{2}\right|$. It implies that $V_{2}^{\prime}$ has size at least $\varrho \cdot\left|V_{2}\right| / 2$. Consequently, the throughput $\tau^{\prime}$ generated by partition $V_{1}^{\prime}, V_{2}^{\prime}, \cdots, V_{k}^{\prime}, \cdots, V_{k_{2}}^{\prime}$ is at least $\varrho \cdot\left(\frac{\left|V_{1}\right|}{2}+\frac{\left|V_{2}\right|}{2 \cdot 2^{2}}\right)$. Remember that the canonical coloring has throughput $\tau$ at most $\frac{\left|V_{1}\right|}{2}+2 \cdot \frac{\left|V_{2}\right|}{2^{2}}$ using fact $\left|V_{i}\right| \leq\left|V_{2}\right|$. From $\left|V_{2}\right| \leq\left|V_{1}\right|$, it is easy to show that $\tau^{\prime} \geq \frac{5}{8} \varrho \cdot \tau$. This finishes the proof.

\section{Approximation Algorithms For Interference Graphs}

Throughout of this section, we use $V$ to denote the set of given radio nodes. All nodes in $V$ are assumed to locate in a plane. The transmission radius of For each node $v \in V$, its transmission radius is denoted by $r_{v}$. The nodes in $V$ are said to have quasi-uniform transmission radii if the ratio of $\max _{v \in V} r_{v}$ to $\min _{v \in V} r_{v}$ is at most $\frac{1}{2 \sin \frac{360^{\circ}}{13}}$, and have uniform transmission radii is all $r_{v}$ 's are equal. We use $G$ to denote the interference graph.

\subsection{First-Fit Prefix-Free Coloring}

First-fit coloring is a class of greedy algorithms for conventional (proper) vertex coloring. Each first-fit coloring is associated with a vertex ordering and colors the vertices sequentially according to the associated vertex ordering by assigning each vertex the least possible color. A first-fit coloring of a graph $G$ using $k$ colors partitions $V$ into $k$ independent sets $V_{1}, V_{2}, \cdots, V_{k}$ where $V_{i}$ is the set of vertices receiving the $i$-th color. Note that $V_{1}$-the set of vertices receiving the first (smallest) color- is always a maximal independent set. In addition, for any $1 \leq i<j \leq k$, at least one vertex in $V_{j}$ is adjacent to some vertex in $V_{i}$.

A first-fit coloring can be adapted for max-throughput prefix-free coloring in the following "unbalanced" manner. First apply the first-fit coloring to obtain a proper vertex coloring. Assume that $k$ colors are used. Replace the $i$-th color by the binary color $1^{i} 0$ for $1 \leq i \leq k-1$, and replace the $k$-th color by the binary color $1^{k}$. Such prefix-free coloring is referred to as unbalanced first-fit prefix-free coloring. 
A first-fit coloring can also be adapted for max-bottleneck prefix-free coloring in the following "balanced" manner. First apply the first-fit coloring to obtain a proper vertex coloring. Assume that $k$ colors are used. Let $T_{k}$ be a balanced full binary tree of $k$ leaves. By mapping the root of $T_{k}$ to the binary color 1 , the $k$ leaves of $T_{k}$ correspond to $k$ binary colors $c_{1}, c_{2}, \cdots, c_{k}$ in the increasing order. For each $1 \leq i \leq k$, replace the $i$-th color in the first-fit coloring by the binary color $c_{i}$. Such prefix-free coloring is referred to as balanced first-fit prefix-free coloring.

As with first-fit coloring, the performance of a first-fit prefix-free coloring depends on the associated vertex ordering. In this paper, we consider the following three vertex orderings:

1. Radius-increasing ordering: In this ordering, the vertices are sorted in the increasing order of their transmission radii.

2. Radius-decreasing ordering: In this ordering, the vertices are sorted in the decreasing order of their transmission radii.

3. Lexicographic ordering: In this ordering, the vertices are sorted in the lexicographic order of their coordinates.

We propose unbalanced first-fit prefix-free coloring in radius-increasing ordering as a heuristic for max-throughput prefix-free coloring. Its performance is given in the following theorem.

Theorem 7 Unbalanced first-fit prefix-free coloring in radius-increasing ordering is a 26-approximation for max-throughput prefix-free coloring. If all nodes have quasi-uniform transmission radii, then it is a 24-approximation for max-throughput prefix-free coloring.

Proof. Let $V_{1}$ be the set of vertices receiving the binary color 10. It was proved in [23] that $\left|V_{1}\right| \geq$ $\alpha(G) / 13$. Thus, the throughput of the output prefix-free coloring is at least $\left|V_{1}\right| / 2 \geq \alpha(G) / 26$. By Theorem $4, \alpha(G) \geq \tau(G)$. Thus, the throughput of the output prefix-free coloring is at least $\tau(G) / 26$. This implies that unbalanced first-fit prefix-free coloring in radius-increasing ordering is a 26-approximation for max-throughput prefix-free coloring.

If all nodes have quasi-uniform transmission radii, then it was proved in [23] that $\left|V_{1}\right| \geq$ $\alpha(G) / 12$. Using the same argument as in the previous paragraph, we can show that in this case unbalanced first-fit prefix-free coloring in radius-increasing ordering is a 24-approximation for max-throughput prefix-free coloring.

We propose balanced first-fit prefix-free coloring in radius-decreasing ordering as a heuristic for max-bottleneck prefix-free coloring. The following theorem gives an upper bound on its approximation ratio.

Theorem 8 Balanced first-fit prefix-free coloring in radius-decreasing ordering is a 16-approximation for max-bottleneck prefix-free coloring.

Proof. Let $k$ be the number of binary colors used by the output prefix-free coloring. Then the number of bits in any of these $k$ binary colors is at most $1+\lceil\log k\rceil$. The bottleneck of the output prefix-free coloring is at least $2^{-\lceil\log k\rceil}$. It was proved in [23] that $k \leq 13 \chi(G)$. By Theorem 4 , the bottleneck of the output prefix-free coloring is at least

$$
\begin{aligned}
2^{-\lceil\log (13 \chi(G))\rceil} & \geq 2^{-\lceil\log 13\rceil-\lceil\log \chi(G)\rceil} \\
& =2^{-\lceil\log \chi(G)\rceil} / 16 \\
& =\beta(G) / 16 .
\end{aligned}
$$


This implies that balanced first-fit prefix-free coloring in radius-decreasing ordering is a 16-approximation for max-throughput prefix-free coloring.

When all nodes have uniform transmission radii, we propose unbalanced first-fit prefix-free coloring in lexicographic ordering as a heuristic for max-throughput prefix-free coloring, and balanced first-fit prefix-free coloring in lexicographic ordering as a heuristic for max-bottleneck prefix-free coloring. Their performances are given in the following theorem.

Theorem 9 Assume all nodes have uniform transmission radii. Then unbalanced first-fit prefixfree coloring in lexicographic ordering is a 14-approximation for max-throughput prefix-free coloring, and balanced first-fit prefix-free coloring in lexicographic ordering is an 8-approximation for maxbottleneck prefix-free coloring.

Proof. Let $V_{1}$ be the set of vertices receiving the binary color 10 in the output of unbalanced first-fit prefix-free coloring in lexicographic ordering. It was proved in $[23]$ that $\left|V_{1}\right| \geq \alpha(G) / 7$. Following the same argument as in the proof of Theorem 7, unbalanced first-fit prefix-free coloring in lexicographic ordering is a 14-approximation for max-throughput prefix-free coloring.

Let $k$ be the number of binary colors used by the output of balanced first-fit prefix-free coloring in lexicographic ordering. It was proved in [23] that $k \leq 7 \chi(G)$. Following the same argument as in the proof of Theorem 8, we can show that balanced first-fit prefix-free coloring in lexicographic ordering is an 8-approximation for max-throughput prefix-free coloring.

We observe that an unbalanced first-fit prefix-free coloring achieves a good throughput but a very poor bottleneck. Indeed, every unbalanced first-fit prefix-free coloring always outputs an extremely unbalanced coloring with colors correspond to the leaves of the binary tree depicted in Figure 4 (a). On the other hand, a balanced first-fit prefix-free coloring achieves a good bottleneck but may have a poor throughput. In the next, we discuss on how to modify them so as to achieve both good throughput and good bottleneck.

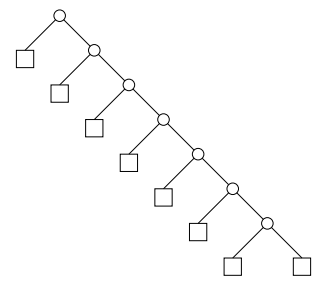

(a)

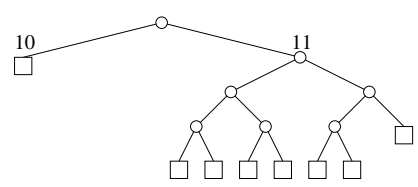

(b)

Figure 4: Modification to the coloring by first-fit: (a) the original colors; (b) the new colors.

For disparate transmission radii, the modified first-fit prefix-free coloring consists of two steps. In the first step, we apply the first-fit heuristic in the radius increasing ordering to find a maximal independent set. All nodes in the obtained maximal independent set will receive the binary color 10. This first step ensures a good throughput. In the second step, we use the first-fit coloring in the radius decreasing ordering to find a proper vertex coloring of the remaining nodes. These colors will then be mapped to the binary colors which correspond to the leaves of a balanced full binary tree rooted at the color 11 (see Figure 4 (b)). This second step ensures a good bottleneck. Such modified first-fit prefix-free coloring is referred to as bicriteria first-fit prefix-free coloring in double radius-ordering. Its performance is given in the following theorem. 
Theorem 10 Bicriteria first-fit prefix-free coloring in double radius-ordering is a 26-approximation for max-throughput prefix-free coloring and a 32-approximation for max-bottleneck prefix-free coloring. If all nodes have quasi-uniform transmission radii, then it is a 24-approximation for maxthroughput prefix-free coloring and a 16-approximation for max-bottleneck prefix-free coloring.

The proof of Theorem 10 is similar to those of Theorem 8 and Theorem 7 and is omitted here.

For uniform transmission radii, we modify first-fit prefix-free vertex coloring in lexicographic ordering as follows: We first apply the first-fit in lexicographic ordering to find a proper vertex coloring. Then the smallest color is mapped to the binary color 10, and all other colors are mapped to the binary colors which correspond to the leaves of a balanced full binary tree rooted at the color 11 (see Figure 4 (b)). Such modified first-fit prefix-free coloring is referred to as bicriteria first-fit prefix-free coloring in lexicographic ordering. Its performance is given in the following theorem.

Theorem 11 Assume all nodes have uniform transmission radii. Then bicriteria first-fit prefixfree coloring in lexicographic ordering is a 14-approximation for max-throughput prefix-free coloring and a 16-approximation for max-bottleneck prefix-free coloring.

The proof of Theorem 11 is similar to that of Theorem 9 and is omitted here.

\subsection{Tile Prefix-Free Coloring}

In this subsection, we assume that all nodes have uniform transmission radii equal to one. We propose a spatial divide-and-conquer heuristic referred to as tile prefix-free coloring. It is attractive due to its easy implementation, especially for dynamic and on-line prefix-free coloring and also distributed prefix-free vertex coloring.

In this heuristic, we tile the plane into regular hexagons of side equal to $1 / 2$ (see Figure 5). Each hexagon, or cell, is considered to be left-closed and right-open, with the top-most point included and the bottom-most point excluded (see Figure 6). Cells are further grouped into clusters of size 12 according to the pattern as shown in Figure 5. We then label the 12 hexagons in a cluster with the numbers 1 through 12 in an arbitrary pattern, and repeat the same labelling for all clusters. Then, the distance between any two (half-closed and half-open) hexagons with the same label is greater than 2. Thus, colors can be spatially reused among the hexagons with the same label.

Now for each $1 \leq i \leq 12$, let $V_{i}$ denote the set of nodes within the hexagons labelled with $i$. We will assign colors to the nodes such that for any $1 \leq i<j \leq 12$, the colors assigned to nodes in $V_{i}$ are disjoint from the colors assigned to nodes in $V_{j}$. For this purpose, all nodes in a set $V_{i}$ will receive colors which are descendants of some color $c_{i}$ corresponding to a leaf in the balanced full binary tree with 12 leaves as shown in Figure 7. For each $V_{i}$, we further partition into groups such that each group consists of nodes in $V_{i}$ that are within a hexagon. Since the interference graph over all nodes in a group is a clique, we apply a "shifted-down" version of the algorithm for prefix-free vertex coloring of complete graphs to all nodes in a group. In other words, the coloring to nodes in each group of $V_{i}$ corresponds to a balanced full binary tree rooted at $c_{i}$ with one-to-one correspondence between the nodes and the leaves. With this coloring, the throughput of all nodes in a group of $V_{i}$ is exactly the rate of $c_{i}$. Thus, in order to maximize the throughput, the mapping from $V_{i}$ 's to $c_{i}$ 's are chosen such that a set $V_{i}$ with more groups will be mapped to a color $c_{i}$ of shorter length.

The next theorem give the performance of title prefix-free coloring. 


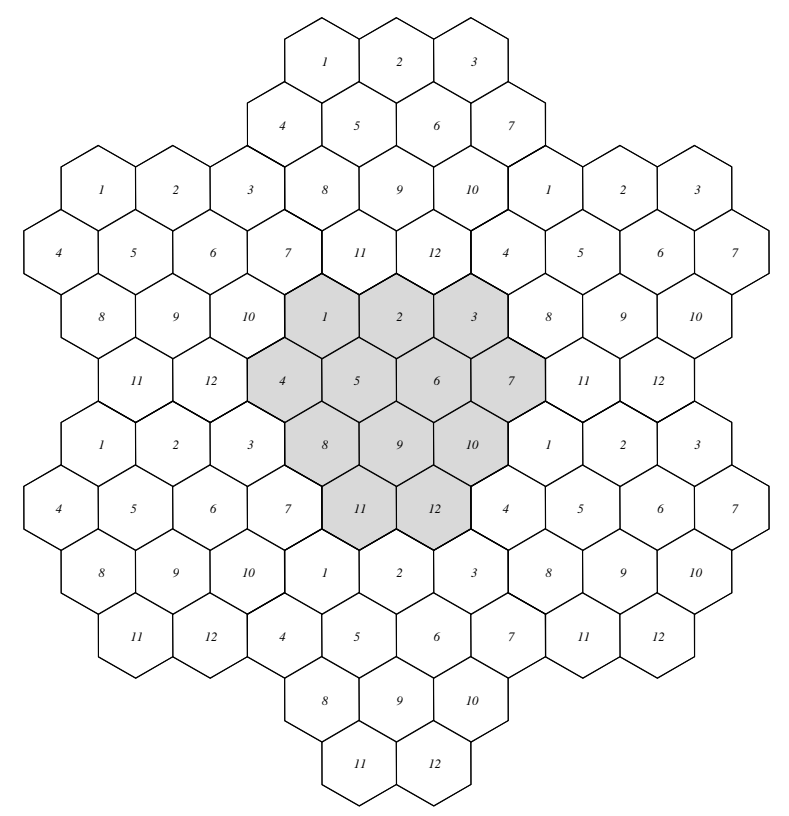

Figure 5: Tiling of the plane into hexagons with 12 hexagons per cluster.

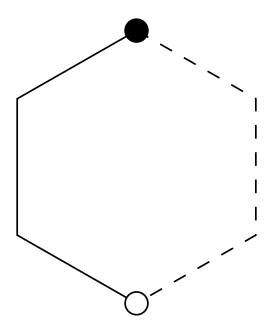

Figure 6: Half-closed half-open hexagon.

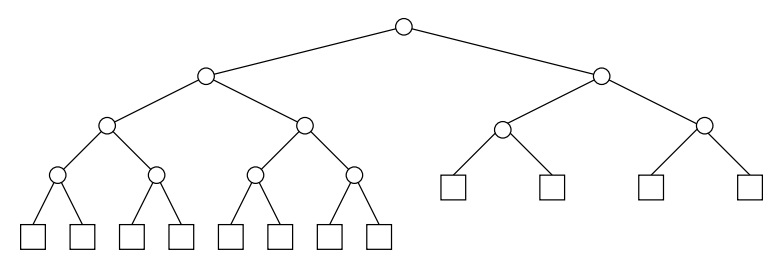

Figure 7: Each of the 12 colors corresponding to the 12 leaves is the prefix of the colors assigned to all nodes in some $V_{i}$. 
Theorem 12 Assume all nodes have uniform transmission radii. Then tile prefix-free coloring is a 12-approximation for max-throughput prefix-free coloring and a 16-approximation for max-bottleneck prefix-free coloring.

Proof. We first prove that tile prefix-free coloring is a 12-approximation for max-throughput prefix-free coloring. For each $1 \leq i \leq 12$, let $g_{i}$ denote the number of hexagons labelled with $i$ which contains at least one node. Note that in any prefix-free coloring the total rates of the binary colors assigned to all nodes in a non-empty hexagon is at most one. Thus,

$$
\tau(G) \leq \sum_{i=1}^{12} g_{i}
$$

Without loss of generality, assume that

$$
g_{1} \geq g_{2} \geq \cdots \geq g_{12} .
$$

Since in tile prefix-free coloring the total rates of binary colors assigned to all nodes in a non-empty hexagon labelled with $i$ is exactly the rate of the binary color $c_{i}$, the throughput of tile prefix-free coloring is exactly

$$
\frac{1}{8} \sum_{i=1}^{4} g_{i}+\frac{1}{16} \sum_{i=5}^{12} g_{i}
$$

Note that

$$
\begin{aligned}
\left(\frac{1}{8} \sum_{i=1}^{4} g_{i}+\frac{1}{16} \sum_{i=5}^{12} g_{i}\right)-\frac{1}{12} \sum_{i=1}^{12} g_{i} & =\frac{1}{24} \sum_{i=1}^{4} g_{i}-\frac{1}{48} \sum_{i=5}^{12} g_{i} \\
& \geq \frac{1}{24} \cdot 4 g_{4}-\frac{1}{48} \cdot 8 g_{5} \\
& =\frac{g_{4}-g_{5}}{6} \\
& \geq 0 .
\end{aligned}
$$

Therefore,

$$
\frac{1}{8} \sum_{i=1}^{4} g_{i}+\frac{1}{16} \sum_{i=5}^{12} g_{i} \geq \frac{1}{12} \sum_{i=1}^{12} g_{i} \geq \frac{1}{12} \tau(G) .
$$

This implies that tile prefix-free coloring is a 12-approximation for max-throughput prefix-free coloring.

Next, we prove that tile prefix-free coloring is a 16-approximation for max-bottleneck prefix-free coloring. Let $m$ be the largest number of nodes contained in a hexagon. Then each binary color used in tile prefix-free coloring has at most $5+\lceil\log m\rceil$ bits. Thus, the bottleneck of tile prefix-free coloring is at least $2^{-4-\lceil\log m\rceil}$. On the other hand, $\chi(G) \geq m$. Thus, by Theorem 4 ,

$$
\beta(G)=2^{-\lceil\log \chi(G)\rceil} \leq 2^{-\lceil m\rceil} .
$$

So the bottleneck of tile prefix-free coloring is at least

$$
2^{-4-\lceil\log m\rceil} \geq \frac{1}{16} \beta(G) .
$$

This implies that tile prefix-free coloring is a 16-approximation for max-bottleneck prefix-free coloring. 


\subsection{Improved Approximation Ratio}

In previous sections, we present several simple heuristics that achieve constant approximation ratios for the throughput and/or the bottleneck of optimal CDMA code assignment for a wireless ad hoc network. These heuristics have the advantage that they could be implemented efficiently and also in a distributed manner. In the remaining of the section, we present a new method with a better approximation ratio. Theorem 6 implies that if we can find a maximal independent set of the interference graph with approximation ratio $\rho$, then we could have a CDMA code assignment method whose throughput is at least $\frac{5}{8} \varrho$ of the optimum. The method will work as follows:

1. Find a maximal independent set, say $V_{1}$, from the interference graph $G$, using an $\rho$-approximation method and then assign CDMA code 10 to every node in $V_{1}$.

2. Remove nodes set $V_{1}$ from $G$ and then find a maximal independent set, denoted as $V_{2}$, from the remaining graph again using a $\rho$-approximation method. Then assign code 110 to every node in $V_{2}$.

3. For the remaining nodes, we could assign CDMA code to them using the first-fit heuristic.

The main task left is to find an algorithm with small approximation ratio for the maximum independent set problem for the interference graph. Li et al. [14] showed that there is a polynomial time approximation scheme (PTAS) for the maximum independent set problem for the interference graph defined here. In other words, given any positive $0<\epsilon<1$, we can find a maximal independent set whose size is at least $1-\epsilon$ times of that of the optimum. Based on the results presented in [14], it is not difficult to show that the time-complexity of the PTAS based on shifting strategy is $O\left(\frac{1}{\epsilon^{2}} n^{O\left(\frac{1}{\epsilon^{4}}\right)}\right)$, where $n$ is the number of wireless nodes. Thus, we have the following theorem

Theorem 13 The throughput of the CDMA code assignment for a wireless ad hoc network can be approximated within $\frac{5}{8}(1-\epsilon)$ in time $O\left(\frac{1}{\epsilon^{2}} n^{O\left(\frac{1}{\epsilon^{4}}\right)}\right)$.

\subsection{A More General Wireless Network Model}

So far we assume that each wireless node $v$ has a transmission range $r_{v}$ such that all nodes inside the disk centered at $v$ using $r_{v}$ as radius will receive the signal from $v$ and this disk contains all possible nodes, to whom the transmission by node $v$ will interfere. In practice, the region where the transmission of node $v$ could interfere is often larger than the region where the transmission of node $v$ could be received correctly. Thus, we study a more general wireless network model where each wireless node $v$ has two different ranges: the transmission range and the interference range. Define the transmission radius of node as the radius of the disk representing its transmission region. Similarly, the interference radius is the radius of the disk representing its interference region. Each node $v \in V$ has a transmission radius $t_{v}$ and an interference radius $r_{v}$. We always assume that $t_{v} \leq r_{v}$.

Let $D(v, r)$ denote the disk centered at $v$ with radius $r$. Then each node defines two disks: the transmission disk $D\left(v, t_{v}\right)$ and the interference disk $D\left(v, r_{v}\right)$. The set of wireless node $V$ defines two sets of disks $\mathcal{T}=\left\{D\left(v, t_{v}\right) \mid v \in V\right\}$ and $\mathcal{D}=\left\{D\left(v, r_{v}\right) \mid v \in V\right\}$ in a two dimensional plane. Given two wireless nodes $u$ and $v$, define their intersection region $I(u, v)$ as $\left(D\left(u, t_{u}\right) \cap D\left(v, r_{v}\right)\right) \cup$ $\left(D\left(u, r_{u}\right) \cap D\left(v, t_{v}\right)\right)$. See Figure 8 for an illustration of intersection regions. Two nodes $u$ and $v$ can communicate with each other directly if they are inside the transmission disk of each other. The transmission a node $u$ interfere with node $v$ if $v$ is inside the interference disk $D\left(u, r_{u}\right)$ of node $u$. Clearly, when $I(u, v)$ is null, nodes $u$ and $v$ can be assigned the same channel since the transmission of one of them cannot interfere with the transmission of the other. Most importantly, 


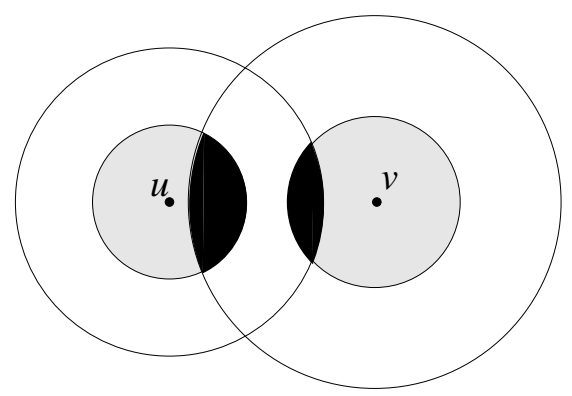

Figure 8: The black region denotes $I(u, v)$. Here the lightly shaded disks denote the transmission regions and the white larger disks denote the interference regions.

when $I(u, v)$ is not null, nodes $u$ and $v$ can still use the same channel if $I(u, v)$ does not contain any other wireless node inside. To capture this property, we define the interference graph $G$ as follows: two nodes $u$ and $v$ are connected in $G$ iff there is a node from $V$ inside $I(u, v)$.

Assume that we know the interference radius $r_{v}$ of each wireless node $v$ and the edge list of the underlying interference graph. If the graph structure is unknown, additionally we need the transmission radius $t_{v}$ and the exact geometry location of each node $v$ to construct it. We then present an efficient method to approximate the maximum independent set, which in turn will give an approximation on the throughput of the CDMA code assignment for this wireless ad hoc network. Our method works as follows.

1. Finds the node with the smallest interference radius $r_{v}$, and adds it to the independent set;

2. Removes this node and all its adjacent nodes from the graph.

3. Repeat the above steps until the graph is empty.

Obviously, this algorithm does compute a maximal independent set with $O(n \log n)$ running time. We then prove the following theorem that guarantees the quality of the computed independent set. We actually show that the interference graph model introduced here have hereditary property: there is a node with a constant bounded number of independent neighbors, and the subgraph by removing this node and its neighbors also does. Let IS be the computed independent set of nodes.

Theorem 14 The computed IS has size at least $\frac{1}{40}$ of that of MIS for the interference graph model defined above.

Proof. We prove this using an area argument. Consider any node $u$ selected by our algorithm. The nodes from an optimum solution, which are connected to $u$ and removed by $u$, can be partitioned into two cases: outside $D_{u}$ or inside $D_{u}$. Remember that here $D_{u}=D\left(u, r_{u}\right)$ is interference disk in our notations.

First consider the nodes outside $D_{u}$. Let $v_{1}, v_{2}, \cdots, v_{k} \notin D_{u}$ be the $k$ nodes from the optimum solution that are connected to $u$ and are removed by the algorithm due to the removing of $u$. The selection of $u$ implies that $r_{v_{i}} \geq r_{u}$ for all $i=1,2, \cdots, k$.

Node $v_{i}, i=1,2, \cdots, k$ is connected to $u$ implies that $D_{i}=D\left(v_{i}, r_{v_{i}}\right)$ intersects with disk $D_{u}=D\left(u, r_{u}\right)$ because if they do not intersect then obviously $I\left(u, v_{i}\right)$ is empty, which further implies that there is no edge $u v_{i}$ in any graph models introduced here. In addition, the disk $D_{i}=D\left(v_{i}, r_{v_{i}}\right)$ centered at $v_{i}, i=1,2, \cdots, k$ cannot contain any node $v_{j}, j \neq i$ inside because all disks centered at $i=1,2, \cdots, k$ are mutually independent in the corresponding interference graph. If $v_{j}$ is inside $D_{i}=D\left(v_{i}, r_{v_{i}}\right)$, then $I\left(v_{i}, v_{j}\right)$ contains $v_{j}$ regardless of the transmission radii of $v_{i}$ and $v_{j}$, implying that $v_{i} v_{j}$ is an edge in the interference graph. 
Let $B_{u}$ be the disk centered at $u$ with radius $2 r_{u}$ and $T_{u}=B_{u}-D_{u}$. Then every disk $D_{i}$, $i=1,2, \cdots, k$, intersect $T_{u}$ since it intersects $D_{u}$. It is not difficult to show that $T_{u} \cap D_{i}$ achieves the smallest area when $v_{i}$ is on the boundary of $B_{u}$ and $r_{v_{i}}=r_{u}$. See Figure 9 . We can show that $\angle w v_{2} u>\frac{5}{12} \pi$. Thus, this smallest area is at least $\frac{5}{12} \pi$. Notice the area of $T_{u}$ is $3 \pi$.
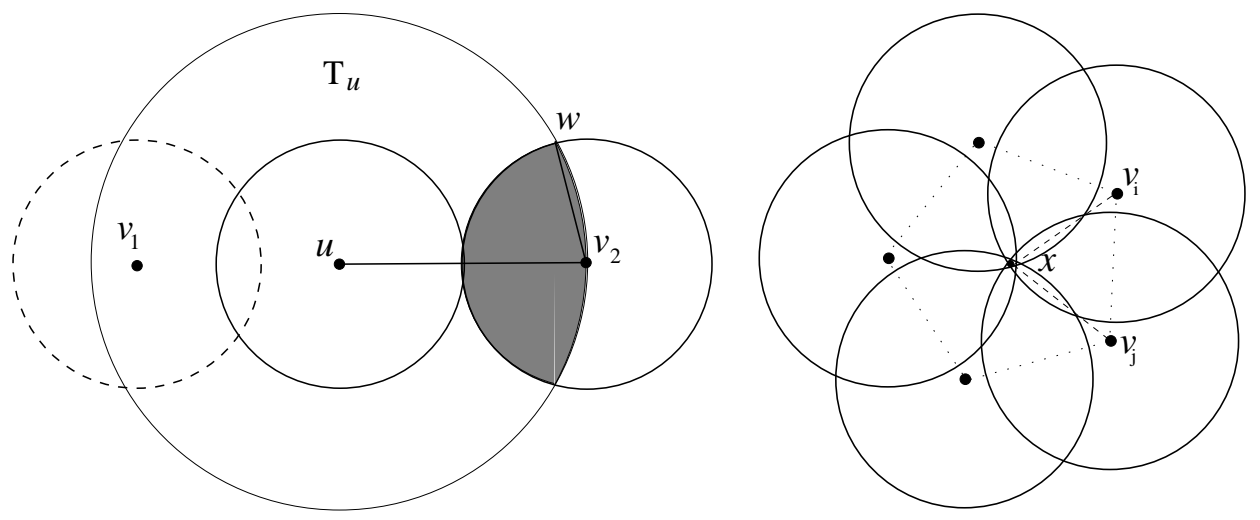

Figure 9: Left: The intersection $T_{u} \cap D_{i}$ is bounded from below. Right: The number of independent disks which cover any point $x$ is bounded by 5 .

Notice that the region $T_{u} \cap D_{i}$ and $T_{u} \cap D_{j}$ for $1 \leq i, j \leq k$ may overlap. However, we will show that every point $x$ is covered by at most 5 disks from $D_{i}, i=1,2, \cdots, k$. See Figure 9. Assume node $x$ is covered by two disks $D_{i}$ and $D_{j}$, i.e., $\left\|x v_{i}\right\| \leq r_{v_{i}}$ and $\left\|x v_{j}\right\| \leq r_{v_{j}}$. Then $\left\|v_{i} v_{j}\right\|>\max \left(r_{v_{i}}, r_{v_{j}}\right)$ because $D_{i}$ and $D_{j}$ are independent in the corresponding interference graph, which implies that $\angle v_{i} x v_{j}>\frac{\pi}{3}$. Thus, $x$ is covered by at most 5 independent disks. Therefore, by an area argument, we have $k \cdot \frac{5}{12} \pi<5 \cdot 3 \pi$. Thus, $k \leq 35$.

Then consider the nodes inside $D_{u}$. Let $v_{1}, v_{2}, \cdots, v_{h} \in D_{u}$ be the $h$ nodes from the optimum solution that are connected to $u$ and are removed by the algorithm due to the removing of $u$. Then obviously, all disks centered at $v_{i}, i=1,2, \cdots, h$ contain node $u$. Since $u$ is covered by at most 5 disks from previous analysis, $h \leq 5$.

Consequently, there are at most $35+5=40$ independent nodes removed when we remove all nodes adjacent to a node $u$ selected by our algorithm. This finishes the proof of our theorem.

Combining Theorem 14 and Theorem 6, we have the following theorem

Theorem 15 The throughput of the OVSF-CDMA code assignment to a wireless ad hoc network where each node has an interference disk and transmission disk can be approximated within 1/64.

We also could design a PTAS for the interference graph of a general wireless ad hoc network model, which is omitted here due to space limit.

\section{Conclusion}

In FDMA, TDMA or OFSF-CDMA wireless ad hoc networks, a conflict-free channel assignment is equivalent to a conventional (proper) vertex coloring of the underlying interference graphs. Because of the limited number of channels available in these networks, the cost metric of a conflict-free channel assignment in these networks is typically the number of channels used. In OVSF-CDMA wireless ad hoc networks, a conflict-free channel assignment is no longer equivalent to a conventional vertex coloring of the underlying interference graphs. Indeed, since not every pair of OVSF 
codewords are orthogonal to each other, the channels assigned to any pair of nodes adjacent to each other in the interference graph must receive not only be different from each other, but also be orthogonal to each other. Because of this constraint, we introduce a new type of vertex coloring called prefix-free (vertex) coloring with positive binary numbers. A conflict-free channel assignment in OVSF-CDMA wireless ad hoc networks is equivalent to a prefix-free coloring of the underlying interference graphs. Furthermore, since there are infinite number of channels in OVSFCDMA wireless ad hoc networks, the number of channels used is no longer an concern. Instead, the throughput and the bottleneck become appropriate cost metrics of a conflict-free channel assignment in OVSF-CDMA wireless ad hoc networks. Correspondingly, we introduced the concepts of the throughput and bottleneck of a prefix-free coloring, and the throughput and bottleneck of a graph. we also introduced two new maximization problems, namely max-throughput prefix-free coloring and max-bottleneck prefix-free coloring

In this paper, we first established two fundamental relations between the independence number and the throughput of a graph, and between the chromatic number and the bottleneck of a graph respectively. After that, we proposed several algorithms for prefix-free coloring. Each of these algorithms is either a constant-approximation for max-throughput prefix-free coloring, or a constant-approximation for max-bottleneck prefix-free coloring, or constant-approximations for both max-throughput prefix-free coloring and max-bottleneck prefix-free coloring at the same time. We leave it as a future work whether we can design a PATS for maximizing the throughput of the OVSF-CDMA code assignment for the inference graph of a wireless ad hoc network.

\section{References}

[1] F. Adachi, M. Sawahashi, and K. Okawa, Tree-structured generation of orthogonal spreading codes with different lengths for the forward link of DS-CDMA mobile radio, IEE Electronics Letters, vol. 33, no. 1, pp. 27-28, January 1997.

[2] T.H. Cormen, C.E. Leiserson, R.L. Rivest, and C. Stein, Introduction to Algorithms (2nd ed.), Cambridge, MA: MIT Press, 2001.

[3] M. Dell' Amico, M.L. Merani, and F. Maffioli, Efficient algorithms for assignment of OVSF codes in wideband CDMA, in Proc. ICC'02, 2002.

[4] A. Bar-Noy, M. Bellare, M.M. Halldórsson, H. Shachnai, and T. Tamir, "On chromatic sums and distributed resource allocation", Inform. and Comput. 140, 183-202, 1998.

[5] R. G. Cheng and P. Lin, OVSF code channel assignment for IMT-2000, IEEE Vehicular Technology Conference, vol. 3, pp. 2188-2192, 2000.

[6] I. Chlamtac and A. Farago, Making Transmission Schedules Immune to Topology Changes in Multi-Hop Packet Radio Networks, IEEE/ACM Transactions on Networking, Vol. 2, No. 1, pp. 23-29, Feb. 1994.

[7] I. Chlamtac and S. Kutten, A spatial reuse tdma/fdma for mobile multihop radio nertworks, in IEEE INFOCOM, pp. 389-394, March 1985.

[8] B.N. Clark, C.J. Colbourn, and D.S. Johnson. Unit disk graphs. Discrete Mathematics, 86:165$177,1990$. 
[9] A. Ephremides and T.V. Truong, Scheduling Broadcasts in Multihop Radio Networks, IEEE Transactions on Communications, vol. 38, no. 4, pp. 456-60, April 1990.

[10] R. Fantacci and S. Nannicini, Multiple access protocol for integration of variable bit rate multimedia traffic in UMTS/IMT-2000 based on wideband CDMA, IEEE Journal on Selected Areas in Communications, vol. 18, no. 8, pp. 1441-1454, August 2000.

[11] J.J. Garcia-Luna-Aceves and J. Raju, Distributed assignment of codes for multihop packetradio networks, in Proc. IEEE MILCOM, 1997.

[12] S.O. Krumke, M.V. Marathe and S.S. Ravi, Models and Approximation Algorithms for Channel Assignment in Radio Networks, Wireless Networks, Vol. 7, Issue 6, Nov. 2001, pp. 567-574.

[13] Ewa Kubicka and A. J. Schwenk, An introduction to Chromatic Sums, in Proc. of ACM Computer Science Conference, pp. 39-45, 1989.

[14] Xiang-Yang Li and Yu Wang. Simple heuristics and PTASs for intersection graphs in wireless ad hoc networks. ACM DialM, 2002.

[15] D. W. Matula and L. L. Beck. Smallest-last ordering and clustering and graph coloring algorithms, Journal of the Association of Computing Machinery, 30(3):417-427, 1983.

[16] T. Minn and K.-Y. Siu, Dynamic assignment of orthogonal variable spreading factor codes in W-CDMA, IEEE Journal on Selected Areas in Communications, vol. 18, no. 8, pp. 1429-1440, August 2000.

[17] R. Nelson and L. Kleinrock, Spatial-TDMA: A collision-free multihop channel access protocol, IEEE Transactions on Communications, vol. 33, no. 9, pp. 934944, Sep. 1985.

[18] S. Ramanathan and E.L. Lloyd, Scheduling algorithms for multi-hop radio networks, IEEE/ACM Transactions on Networking, vol. 1, pp. 166-172, April 1993.

[19] R. Ramaswami and K. K. Parhi, Distributed scheduling of broadcasts in a radio network, in IEEE INFOCOM, pp. 497-504, 1989.

[20] A. Sen and M. L. Huson, A New Model for Scheduling Packet Radio Networks, ACM/Baltzer Journal Wireless Networks, 3 (1997), pp. 71-82.

[21] A. Sen and E. Malesinska, Approximation Algorithms for Radio Network Scheduling, Proceedings of 35th Allerton Coneference on Communication, Control and Computing, Champaign, Illinois, pp. 573-582, October, 1997.

[22] D.S. Stevens and M.H. Ammar, Evaluation of slot allocation strategies for TDMA protocols in packet radio networks, IEEE Military Communications Conference, pp: 835-839, 1990.

[23] P.-J. Wan, C.W. Yi, X. Jia, and D. Kim, Approximation Algorithms for Conflict-Free Channel Assignment in Wireless Ad Hoc Networks, submitted for publication, 2003. 